\title{
Some problems in analysing premixed flames by infrared thermography
}

by B. IMBERT, Y. LE MAOULT, J. QUINARD

Laboratoire de Recherche en Combustion, Université de Provence, Centre St Jérôme, S252, 13397 Marseille cedex 13, France.

\section{Abstract}

Quantitative studies of flames by IR thermography can be achieved if we solve properly two problems. At first, there is a low thermal level but corresponding to very high temperatures at which calibration curves are not available, therefore we are concerned with the question of the extrapolability of these curves. Then burned gases are semi-transparent media so that we have to account for 3D effects. Our approach is proved by comparing thermal images of premixed flames in simple configurations and the images calculated by simulation of the complete acquisition system.

\section{List of symbols}

$A, B, C$ : coefficients of the calibration curve;

$D \quad$ : distance of observation;

$D_{p} \quad$ : pupil diameter;

d : slit width in mrd;

$\delta \quad:$ distance to the plane of focus;

G : proportionality coefficient (eq.3);

$h \quad$ : V-flame thickness;

H : vertical distance to the top of the field of view;

$l_{B} \quad$ : background level;

$I_{S} \quad$; slit level;

$\Phi$ : equivalent source diameter in the plane of focus whose image coincides with the blurred image of an out of focus source point;

$K \quad:$ mean absorption coefficient of burned gases;

$L(x) \quad$ : horizontal line profile;

$\lambda \quad$ : wavelength of IR radiation;

$\left.L_{C N} \lambda, T_{C N}\right)$ : monochromatic intensity of black body radiation at temperature $T_{C N}$;

$N \quad$ : detector output voltage in binary levels;

$N^{\prime}::=\mathrm{d} N / \mathrm{d} T$

$N(h) \quad$ : vertical line profile in binary levels;

$N_{b} \quad$ : background level of flame;

$N_{F} \quad$ : binary level corresponding to an infinite slab of burned gases;

$P \quad$ : distance of the plane of focus to the camera lens;

$\Pi_{d} \quad$ : slit function of width $d$;

$R(\lambda) \quad$ : relative spectral response of the camera;

$S_{p} \quad$ : pupil surface of the camera;

$S_{\text {Ifov }}$ : equivalent surface of the instantaneous field of view;

$\sigma_{x} \quad$ : characteristic width of the line impulse function in mrd;

$T \quad$ : temperature in Kelvin;

$\tau\left(\lambda, T_{\text {amb }}\right):$ atmospheric spectral transmissivity at temperature $T_{a m b} ;$

$x \quad$ : horizontal abscisse in mrd;

\section{Introduction}

Infrared thermography and pyrometry are widely used in characterizing combustion systems or aircraft engines [1 to 4] and, moreover, it can be a very attractive tool for combustion studies 
since it is non intrusive and has fast enough response time such that field or line informations can be accessed. Recent developments in data acquisition and processing allows for considering infrared cameras as true measurement devices [5], but with further needs in data analyses.

Combustion experiments involve semi-transparent media at very high temperatures setting two main problems: temperature calibrations are not always possible and the thermal image of an elementary source point evolves strongly with its distance to the plane of focus. The first point is analysed from black body radiation measurements between $400 \mathrm{~K}$ and $1100 \mathrm{~K}$ showing the evolution of the classical calibration parameters with the mean temperature. Then the thermal image of an out of focus object is simply calculated by determining in what way it is imaged on the detector, this approximation beeing valid as long as no field diaphragm interferes. It is thus possible to modelize the image resulting from the observation of any distribution of radiative sources.

Concerning the analysis of premixed flames, the thermal scene is easily determined since the burned gases volume is clearly demarcated and its composition and temperature are precisely known from the initial mixture fractions of fuel, oxygen and nitrogen. However the spectral emissivity of the products has to be evaluated from band models which are not so accurate especially in the 8-12 $\mu \mathrm{m}$ long wave domain. It is thus not surprising that large discrepancies appear between experiments and direct simulations in so far as the relative spectral response of the camera is not precisely known. But the general trends are faithfully set out and global parameters or curves of growth can be obtained from such experiments.

\section{Experimental set-up}

The combustion facility is a laminar burner with a section of $10 \times 10 \mathrm{~cm}^{2}$ [6]. The combustible gas is a propane-air mixture whose composition and mass flow rate are controlled by using pressure regulators and calibrated sonic throats. The flame is anchored on a heated rod so that it has a dihedral shape which can be measured by using laser tomography [7] (figure 1). The fresh gases are seeded with oil droplets that scatter the light from a plane laser sheet. The burned gases are thus limitated to the inner part of the V-shape. It has been checked by thermocouple measurements that the mean temperature is constant everywhere in the burned gases contributing to IR radiation (the emissivity of fresh gases is quite negligible). When recording the flame thermograms, a large water cooled flat plate, covered with a large emissivity black paint, is placed $50 \mathrm{~cm}$ behind the burner to reduce ambient contribution to a uniform base level.

The thermographic apparatus is composed of an infrared camera AGEMA 780 LW/SW whose detector output is directly digitized on 12 bits and stored on a PC/AT computer. Thermal calibrations and slit response functions are obtained with a $\mathrm{HGH}-\mathrm{RCN} 900$ black body fixed on an optical bench with micrometric displacements. Each device is controlled by the micro computer allowing for automatic sequences of acquisition.

\section{Temperature calibrations}

The problem here is to relate the output voltage of the detector to the physical quantity of interest in the thermal scene, generally the temperature. Combustion products reach temperatures of about $2000 \mathrm{~K}$ which are not accessible with standard commercial black bodies. Moreover the emissivity spectrum of burned gases is restricted to some discret bands so that it is worthwhile taking cares of the spectral response of the complete system.

Twelve series of temperature calibrations were recorded to point out the reproductibility of such measurements and the influence of various factors: ambient temperature, scanning with increasing or decreasing temperatures to verify the performances of the stabilizing loop, 
operator... The output voltage is expressed in binary levels averaged on 16 consecutive frames and an hundred pixels in the homogeneous area corresponding to the black body center. The results plotted on figure 2 are properly distributed along a continuous curve whose shape is well approximated by the classical relation derived from the Planck's equation:

$$
N=A /[C \exp (B / T)-1]
$$

In this formula, $A$ and $B$ correspond to the physical coefficients of the Planck's law whereas $C$ is only a weighting parameter to account for the integration on a relatively large spectral domain. All these parameters are thus expected to vary with the mean wavelength of the collected radiation which is also a function of the black body temperature according to Wien's law. It is confirmed by fitting this set of parameters on restricted temperature ranges (Table I) so that such curve can't be extrapolated far outside the initial domain of calibration.

Another way of approaching the question of extrapolability is to linearize the relation (1). Introducing the derivative $N^{\prime}=\mathrm{d} N / \mathrm{d} T$, a straightforwards calculation leads to an equivalent equation:

$$
\operatorname{Ln}\left(T^{2} N^{\prime} / N^{2}\right)=\operatorname{Ln}(C B / A)+B / T
$$

The experimental points plotted on figure 3 move apart from the expected straight line but with a smoother variation than on the direct plot so that the mean coefficients $(A, B, C)$ themselves can be extrapolated slightly further. However both approaches fail in determining the calibration curves at typical combustion temperatures.

To rely the output signal of the detector to the IR emission from burned products, it is thus necessary to calculate exactly the electromagnetic flux integrated by the detector. Using the standard relative spectral response of the camera $R(\lambda)$, this can be expressed as:

$$
N=G\left(S_{\text {IFOV }} S_{p} / D^{2}\right) \int R(\lambda) \tau\left(\lambda, T_{\text {amb }}\right) L_{C N}\left(\lambda, T_{C N}\right) d \lambda
$$

where $S_{I F O V}$ scales the effective surface contributing to the signal, $S_{p}$ represents the pupil surface collecting the radiation, $D$ is the distance of observation, $\tau\left(\lambda, T_{\text {amb }}\right)$ the atmospheric spectral transmittivity and $L_{C N}\left(\lambda, T_{C N}\right)$ is the monochromatic intensity of black body radiation at $T_{C N}$. $G$ is a proportionality coefficient related to the electronic conditioning of the detector which has been measured by calculating the integral in the right hand side of equation (3) for a large temperature range (figure 4). This time the relation is very linear so that the binary level can be calculated once we know the effective flux entering the camera.

\section{Imaging of an out of focus radiative source}

This analyse is performed through measurements of the slit response function approaching the line impulse function. As a first approximation this one can be considered Gaussian so that it is only characterized by one parameter $\sigma_{x}$. The line profile $L(x)$ corresponding to a slit of width $d$ can thus be expressed as a convolution product:

$$
L(x)=\left[1 / \sigma_{x} \operatorname{sqrt}(\pi)\right]\left[I_{B}+I_{S} \Pi_{d}(x)\right]^{*}\left[\exp \left(-x^{2} / \sigma_{x}^{2}\right)\right]
$$

$I_{B}$ and $I_{S}$ are respectively the background level and the level corresponding to a large slit or the black body level. The way by which $\sigma_{x}$ can be calculated has been presented in a previous communication [8] and it is only necessary here to see the result (figure 5-a) confirming that the line profile is well reconstructed with this equation. If the slit is translated backwards or forwards the plane of focus, the main resulting effect is that each source point will have a blurred image in the detector plane so that it will be seen as a homogeneous disc whose diameter $\Phi$ in the plane of focus is simply given by geometrical relations:

$$
\Phi=D_{\rho}[\operatorname{abs}(\delta) /(P-\delta)]
$$

where $D_{\rho}$ is the pupil diameter, $P$ the distance of the plane of focus to the camera lens and $\delta$ the distance between the source point and the plane of focus. The scene effectively viewed by 
the camera is thus the convolution product of the actual signal by a spreading function of width $\Phi$. Considering again a slit function, the resulting line profile will be:

$$
L_{\delta}(x)=\left[1 / \sigma_{x} \text { sqrt }(\pi)\right]\left[I_{B}+I_{S} \Pi_{d}(x)\right]^{*}\left[\Pi_{\Phi}(x)\right]^{*}\left[\exp \left(-x^{2} / \sigma_{x}^{2}\right)\right]
$$

It has been verified that such equation describes very well the experimental line profiles (figure5-b) as long as the rate of defocusing $\delta / P$ does not exceed $10 \%$ that is sufficient to simulate most of the usual configurations in IR thermography.

\section{Comparison between numerical simulations and experimental thermograms of V-flames}

The next step to calculate the resulting thermal image of a premixed flame is to determine the emissivity of an elementary cell of burned gases. In a first approach, we only consider IR emission from $\mathrm{CO}_{2}$ and $\mathrm{H}_{2} \mathrm{O}$. Their partial pressures are given from the initial mixture composition (propane-air) and the temperature is calculated from the effective heat of combustion of this mixture [9]. The spectral emissivity of the products is then calculated by using a wide band model $[10,11]$ since it is necessary to describe the whole domain of wavelengthes where the camera is sensitive $(2 \ldots 15 \mu \mathrm{m})$.

To have well defined geometrical and physical properties of the emissive volume, the $V$-flame is observed perpendicularly to the anchoring rod, the lines of the thermal image being parallel to this rod. In this way, there is no net effect of the line impulse function since the thermal scene is very homogeneous in this direction, but defocusing effects are responsible of the spreading of the most external cells by a factor 1.3. A vertical line profile will thus represent the integrated IR radiation as a function of the burned gases thickness.

The comparison between experimental vertical line profiles and direct simulations shows large discrepancies (figure 6) which are not very surprising: the roughness of the wide band models is well known and moreover we only use typical values of the spectral response of the camera so that large errors can result from slight shifts in cut-off frequencies of the main and secondary transmitting windows. However the main features are well described. On the same figure we also report simulated vertical line profiles calculated by fitting a mean value of the integrated emissivity of burned gases. These curves are obviously much closer to the experimental profiles but they exhibit a systematic under-estimation of the thermal signal. This is best shown by adjusting the experimental line profiles to a general function minimizing the initial hypotheses:

$$
N(h)=N_{b}+N_{F}[1-\exp (-K h)]
$$

where $N_{b}$ represents a base level, $N_{F}$ the integrated intensity for an infinite optical thickness and $\mathrm{K}$ a mean integrated absorption coefficient. Such equation fits very well the experimental datas (figure 7) but with unexpected parameters values:

- the base level is much larger than the background plate level, indicating either the existence of some reflection on this plate or the contribution of CO emission (2.35 and $4.7 \mu \mathrm{m})$ which should be relatively uniform all along the flame [12];

- the integrated intensity $N_{F}$ is much smaller than the calculated value at typical combustion temperatures; this can be an artefact from the fitting procedure which picks up the most sensitive part of the absorption phenomenum so that the integrated values correspond to restricted spectral domains: the almost saturated lines and the very weak lines introduce only small changes of the total IR radiation with a next increase in the optical thickness so that their contributions are drowned in the background level; in the same vein the value of $K$ seems sensible but it represents an averaged value on the wavelengthes domain weighted by the spectral response of the camera and on the optical path lengthes so that it can't be analysed without further investigations. 


\section{http://dx.doi.org/10.21611/qirt.1992.051}

\section{Conclusions}

IR thermography of flames or across flames can thus be performed with some special cares. Temperature calibrations can't be extrapolated far outside the initial domain of measurements, but the linear response of the detector to the integrated radiative flux overcomes this difficulty. Blurred images can be calculated very accurately to take into account 3D effects always present in semi-transparent media. However both emissivity spectra of burned gases and the spectral response of the camera have to be precisely known if absolute measurements are needed. To this end, such experiments on well controlled V-flames observed with accurate spectral filters can be used to check spectroscopic models.

\section{REFERENCES}

[1] LAFOLETTE (R.M.), HEDMAN (P.O.) and SMITH (P.J.).- "An analysis of coal particle temperature measurements with two color optical pyrometers", Comb. Sci. Techn., 66, 1989, p .93-105.

[2] BREWSTER (Q.), TAYLOR (D. M.).- "Radiative properties of burning aluminium droplets", Comb. Flame, 72,1988, p. 287-299.

[3] BEDAT (B.), GIOVANNINI (A.) and PAUZIN (S.).- "Instantaneous temperature profile measurements in a flame by infrared line thermometry technique", séminaire EUROTHERM $n^{\circ} 17$, Cascais, Portugal ,1991.

[4] EDWARDS (D. K.), BABIKIAN (D. S.).- "Radiation from a nongray scattering, emitting and absorbing solid rocket motor plume", J. Thermophys. Heat Transfer, 4, n4,1990,p. 446-453.

[5] IMBERT (B.), LABROSSE (G.) and MILLET (C.).- "La thermographie infrarouge numérisée en temps réel sur 12 bits, un équipement mobile de mesures", revue Mesures, $\mathrm{n}^{\circ}$ 16,1988.

[6] BOYER (L.), QUINARD (J.).- "On the dynamics of anchored flames",Comb.Flame, 82 , 1990, p.51-65.

[7] BOYER (L)- "Laser tomographic method for flame front movement studies", Comb. Flame, 39,1980, p. $321-323$.

[8] LE MAOULT (Y.), IMBERT (B.) and QUINARD (J.).-" Thermographie des flammes accrochées: comparaison expériences simulations", journée d'étude de la SFT sur la Thermographie Infrarouge Quantitative, Paris , 1991.

[9] QUINARD (J.).- thèse d'état, Université de Provence, Marseille ,1984.

[10] ROSENHOW (W. H.), GARTNETT (J. P.) and GARNIC (E. M.).- "Handbook of heat transfer fundamentals", ed Mc Graw Hill, 1985.

[11] LUDWIG (C. B.), MALKMUS (W.), REARDON (J. E.) and THOMSON (J. L.).- "Handbook of infrared radiation from combustion gases", NASA SP 3080, 1970.

[12] BECHTEL (J. H.), BLINT (R. J.), DASH (C. J.) and WEINBERGER (D. A.).- "Atmospheric pressure premixed hydrocarbon air flames, theory and experiment", ComB. Flame, 42, 1981, p. 197-213. 


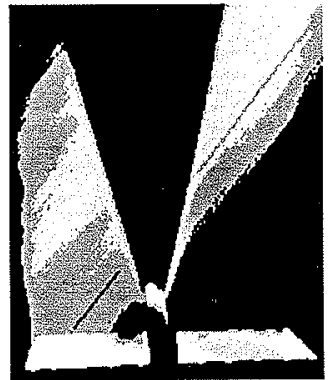

Fig.1- Tomographic cut af anchoredV-flame: propane air equivalence ratio 0.726 ; mean flow speed $2.5 \mathrm{~m} / \mathrm{s}$

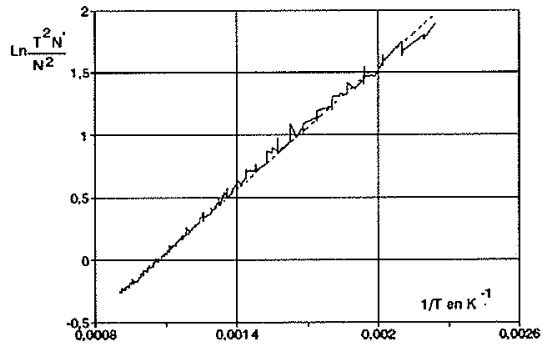

Fig.3- Linearisation of temperature calibration curve (eq.2); dashed line: linear fit with $A=5126, B=1723, C=0.473$

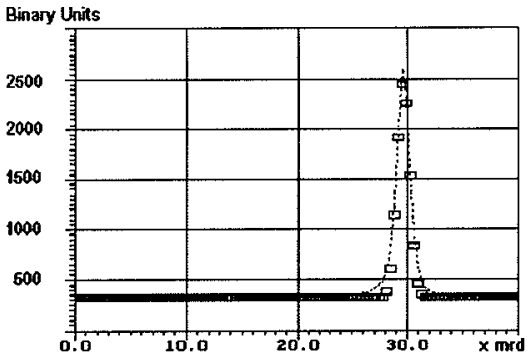

Fig.5a- Comparison between experimental (...) Fig.5b-Comparison between experimental (...) and computed horizontal line profile ( $\square$ ). Focused slit

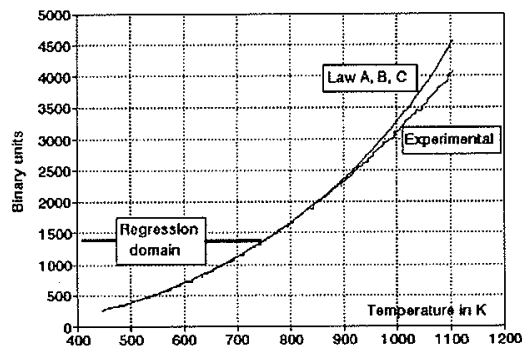

Fig.2- Extrapolability of law A,B,C

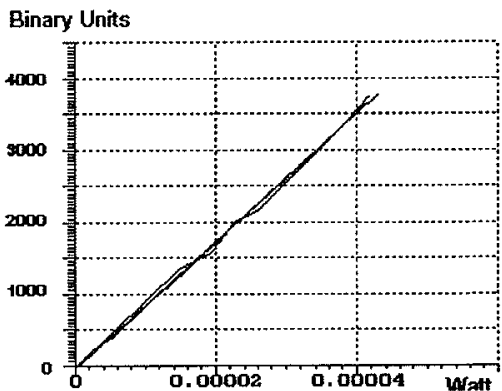

Fig.4- Output of the detector as a function of the power integrated by the camera

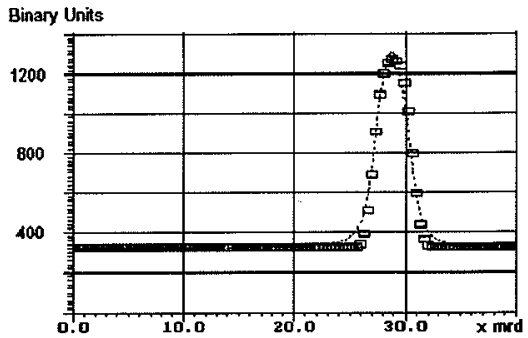

and computed horizontal line profile $(\square)$.

Defocused slit with $\delta / P=5 \%$ 
http://dx.doi.org/10.21611/qirt.1992.051

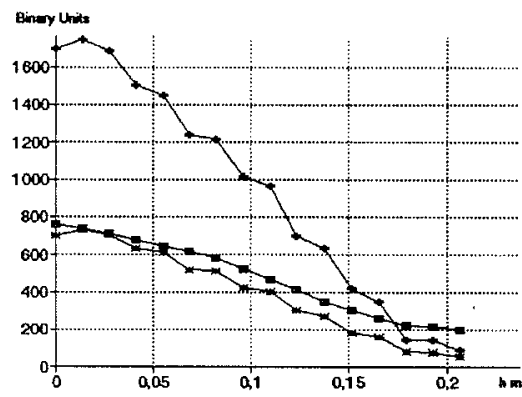

Fig.6- Vertical line profile of a premixed air-propane V-flame; equivalence ratio 0.762 , flow speed $2 \mathrm{~m} / \mathrm{s}$;

(a) experiment ; (+) emissivity 0.0143 ;

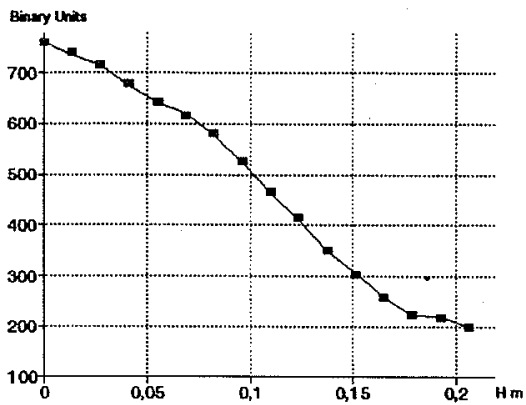

Fig.7- Best fit to experimental vertical line profile (eq. 7): $N_{b}=108, N_{F}=804, K=0.103$

TABLE I

Temperature calibration coefficients as a function of the mean temperature

\begin{tabular}{|c|c|c|c|c|}
\hline$T \dot{m i n}$ & $T \max$ & $A$ & $B$ & $C$ \\
\hline 450 & 1100 & 5130 & 1720 & 0,47 \\
\hline 450 & 775 & 2600 & 1480 & 0,4 \\
\hline 775 & 1100 & 7900 & 1950 & 0,5 \\
\hline 450 & 650 & 3380 & 1540 & 0,45 \\
\hline 500 & 700 & 3780 & 1580 & 0,46 \\
\hline 550 & 750 & 2970 & 1520 & 0,42 \\
\hline 600 & 800 & 3310 & 1570 & 0,42 \\
\hline 650 & 850 & 4800 & 1720 & 0,45 \\
\hline 700 & 900 & 5190 & 1760 & 0,46 \\
\hline 750 & 950 & 6130 & 1830 & 0,47 \\
\hline 800 & 1000 & 7510 & 1930 & 0,49 \\
\hline 850 & 1050 & 9910 & 2060 & 0,53 \\
\hline 900 & 1100 & 9350 & 2040 & 0,52 \\
\hline \hline
\end{tabular}

Standard deviations : $\mathrm{A} \pm 10 \%, \mathrm{~B} \pm 5 \%, \mathrm{C} \pm 2 \%$. 